Gynaecologia 1962;154:377-379

\title{
Index rerum
}

(B) = Buchbesprechung - Book review - Livre nouveau (S) = Gesellschaftsberichte - Society Transactions - Sociétés

Abort, habitueller, männlicher Faktor, 257

-, Statistik, 53

Albino-Ratten, Klimakterium und Menopause, 296

Albuminurie, bei Schwangerschaftsspät-toxikose und Placenta praevia, 1

Amenorrhea, primary, due to intra-uterine adhesions, 181

Aménorrhées, dysménorrhées, 316 (B)

Antigonadotrophins in the suppression of postpartum lactation in non-nursing mothers, 149

Apoplexie utéro-placentaire (Couvelaire) and hydatidiform mole, 213

Asthenospermia, abnormal pregnancy, 321

Azauracilriboside, cancerostatic, 219

Becken, kleines, unklare Schmerzen, Laparoskopie, 367

Blut, Gerinnungsstörungen, während Schwangerschaft und Geburt, 351

-, Gerinnungsverhältnisse des Nabel-venenblutes, 29

Blutung, intraamniale, 120 (S)

Bromelasetest, 124 (S)

Caesarean section, previous, rupture of gravid uterus, 287

Calciumspiegel in graviditate, 114 (S)

Carcinom, Collumcarcinom, Ureterschei-denfistel nach Radikaloperation, 244

-, Gebärmutterkrebs, cytofluoro-mikro-skopische Diagnose, 162

-, Verhornungsindex im Vaginalabstrich, 173

Carcinoma, of the cervix, lymphoangio-graphy, 305 , surgical radicalism, 219

Cardiovascular effects of Syntocinoii, 73

Chirurgie, klinische, für die Praxis, 63 (B)

Chorionic gonadotropin, production, effect of Orga-Steron, 81

Clot-Timer, 124 (S)

Corpus luteum graviditatis, Funktion und Regression, 129

Cytologie, cytofluoromikroskopische Diagnose desGebärmutterkrebses, 162

Dammschnitt, Geburtsvorbereitung, 116 (S)

Desoxyribosenucleinsäure, Gehalt in Spermien, habitueller Abort, 257

Dysménorrhées, aménorrhées, 316 (B)

Eibettstörungen, menschliche Mißbil-dungen, 190 (B)

Eklampsie, toxisches Prinzip in der Placenta, 14

Elektronenmikroskopische Untersuchungen an der Epithel-Bindegewebs-grenze des normalen Portioepithels nach Silbernitratimprägnation, 206

Elektronenoptische Untersuchungen an der glatten Muskulatur des mensch-lichen graviden Uterus, 193 
Endometritis post abortum, 53

Endometrium, Adenocarcinom, 162

Epithel-Bindegewebsgrenze, des normalen Portioepithels, elektronenmikroskopische

Untersuchungen, 206

Fibrinolyse, Blutgerimmngsstörungen während Schwangerschaft und Geburt, 351 , Klinik, 189

(B)

Foetal mortality, vacuum extraction and forceps delivery, 65

Forceps delivery, natal and postnatal mortality, 65

Frau als Mutter, 62 (B)

Frauenheilkunde, Almanach, 316 (B)

Frauenmilch, Einfiüsse langdauernder Laktation, 45

Frühgeborene, Krankheiten, 62 (B)

Frühgeburt, Gerinnungsverhältnisse des Nabelvenenblutes, 29

Funck Brentano, Paul, Prof., 1898-1962. 375

378

Index rerum

Galactopoiesis, suppression, antigonadotrophins, 149 Geburt, Blutgerinnungsstörungen, 351 Geburtshilfe und Gynäkologie, Wandlungen seit der Jahrhundertwende,

93 (S) Geburtsvorbereitung, 116 (S) Gonadotrophines choriales, action inhibitrice sur Гutérus, 272 Gonadotropin, chorionic, production in

early pregnancy, effect of Orga-

Steron, 81 Gynäkologie und Geburtshilfe, Wand-

lungen seit der Jahrhundertwende,

93 (S) Gynecology and clinical obstetrics, 62 (B) -, Novak's textbook, 63 (B) -, some topical

problems, 64 (B) Health and hormones, 192 (B) Herpes, unklare Schmerzen im kleinen

Becken, Laparoskopie, 367 Histochemie, Funktion und Begression

des Corpus luteum graviditatis, 129 Hormones and health, 192 (B) Humanité et subsistance, 315

(B) Hydatidiform mole complicated by apo-

plexie utéro-placentaire (Couvelaire),

213 Hypocalcämie, in graviditate, 114 (S) Hysterectomy, carcinoma of the cervix,

219 íntersexualität, 190 (B) Intraamniale Blutung, 120 (S) Intra-uterine adhesions, primary ame-

norrhea, 181 Kropf, endemischer, Einfluß auf das

Neugeborene, 156 Laboratorium, neuere Methoden und

Apparate, 124 (S) Lactation, postpartum, suppression in

non-nursing mothers, antigonado-

trophins, 149 Laktation, langdauernde, Zusammen-

setzung der Frauenmilch, 45 Laparoskopie, unklare Schmerzen im

kleinen Becken, 367 Leber, Einfluß von Xanthophyll auf die

Speicherung von Vitamin A, 109 (S)

Leucodiff, 124 (S)

Lymphoangiography, cancer of the cervix, 305 Marshall-Marchetti-Operation, vaginale,

105 (S) Milch, langdauernde Laktation und $\mathrm{Zu}-$

sammensetzung der Frauenmilch, 45 Mißbildungen, menschliche, infolge Ei-

bettstörungen, 190 (B) Mole, hydatidiform, 213 Muskulatur, des menschlichen graviden 
Uterus, elektronenoptische Untersu-

chungen, 193 Nabelvenenblut, Germnungsverhält-

nisse, bei Frühgeburten und über-

tragenen Feten, 29 Neugeborenes, Einfluß des endemischen

Kropfes, 156 -, Krankheiten, 62 (B) NeutrAB-Test, 124 (S) Obstetrics, clinical, and gynecology, 62

(B) -, some topical problems, 64 (B) Obstétrique, precis, 317 (B) Oligozoospermia, abnormal pregnancy,

321 Oogenesis, 319 (B) Orga-Steron, effect on the production of

chorionic gonadotropin in early pregnancy, 81 Ovarian tumors, 318 (B) Papanicolaou, Gcorg N., Prof., 1883-

1962, 372 Perinatal mortality, 318 (B) Pipetten, automatische, 124 (S) Pitocin, cardiovascular

effects, 73 Pituitrin, cardiovascular effects, 73 Placenta praevia, Schwangerschafts-

spättoxikosen, 1 -, toxisches Prinzip bei Eklampsie, 14 Portioepithel, Epithel-Bindegewebsgrenze, elektronenmikroskopische Un-

tersuchungen, 206 Pregnancy, abnormal, male factor, 321 -, cardiovascular-renal problems, 318

(B) Pregnyl, action inhibitrice des gonadotrophines choriales sur Гutérus, 272

Index rerum

379

Psychoprophylaxc, Geburtsvorberei-

tung, 116 (S) Radiation therapy, 318 (B) Radikaloperationen, Ureterscheidenfi-

stel, 244 Samen, pathologischer, habitueller

Abort, 257 Schwangerschaft, Blutgerinnungsstö-

rungen, 351 Schwangerschaftsspättoxikose, 1 Schwangerschaftstest,imaiunologiseher,

124 (S) Schwangerschafts-ti bertragung, Gerin-

nungsverhältnisse des Nabelvenen-

blutes, 29 Sectio caesarea, siebenmal in 7 Jahren,

123 (S) Semen, abnormal, pathological development of the fetus, 321 Serumcholinesterase,

Bestimmung, 124

(S) Spättoxikose in der Schwangerschaft,

1 Spektrophotometer Beckman, 124 (S) Spermien, DNS-Gehalt, habitueller

Abort, 257 Stachelzellen, im menschlichen graviden

Uterus, elektronenoptische Untersu-

chungen, 193 Sterilization, prognosis on social-psychiatric grounds, 256 (B) Steroid hormones, mechanism, 315

(B) Stroma, Uterus, Altern, 296 Syntocinon, cardiovascular effects, 73 -, emploi clinique de la tocographie ex-

terne, 334 Teratozoospermia, abnormal pregnancy,

321

Tocographie, externe, emploi clinique, 334

Toxämie, toxisches Prinzip in der Placenta bei Eklampsie, 14

Toxämisches Syndrom, 1

Tumors of the female genitalia, visualization by lymphographic techniques, 305

Ultramikro-Analysesystem, 124 (S)

Ureterscheidenfistel, nach Radikalopera-tion des Collumcarcinoms, 244 
Urology, gynecological, 191 (B)

Uterus, action inhibitrice des gonado-trophines choriales, 272

-, gravid, rupture, 287

-, menschlicher, gravider, elektronenoptische Untersuchungen an der glat-ten Muskulatur, 193

Uterusstroma, Altern, 296

Vacuum extraction, natal and postnatal foetal mortality, 65

Vaginalabstrich, krebskranker Frauen, Verhornungsindex, 173

Vaginale Marshall-Marchetti-Operation, 105 (S)

Vakuumextraktion, 231

Verhornungsindex im Vaginalabstrich krebskranker Frauen, 173

Vitamin A, Speicherung in der Leber von Muttertieren und Foeten, Ein-fluß von Xanthophyll, 109 (S)

Wertheim's operation, cervix carcinoma, 219

- -, Ureterscheidenfistel, 244

$\mathrm{X}$-ray radiation, carcinoma of the cervix, 219

Xanthophyll, und Speicherung von Vitamin A in der Leber von Muttertieren und Foeten, 109 (S)

Index nominum

Abdel-Salam, R., v. Mazhar, K. Althaus, U., v. Neuweiler, W. Alvarez, R. R. de, 318 (B)

Andreoli, C, Vavala, V., and Cavallini,

R., 149 Antener, I., v. Neuweiler, W.

Arneson, A. N., and Nolan, J. F., 318(B)

Berger, J., 206

Berger, M., Neuweiler, W., Richter, R.

H.H. H.

Richterich

R.

Rubinstein

A.,

und Delnon

I.

14 Bergman

P.

and Malmström

$\mathrm{T}$.

65 